\title{
LA 'RED ESPAÑOLA DE AUTORIDADES DE LA COMPETENCIA': LAS POSIBILIDADES DE UN MODELO DESCENTRALIZADO PARA GANAR EN EFICIENCIA
}

José Antonio Rodríguez Miguez

\begin{abstract}
SUMARIO: 1. LAS REDES Y LA APLICACIÓN DESCENTRALIZADA DEL DERECHO DE LA COMPETENCIA. 2. LA INTERNATIONAL COMPETITION NETWORK (ICN): UNA RED DE COMPETENCIA A ESCALA PLANETARIA. 3. LA EUROPEAN COMPETITION NETWORK (ECN) COMO INSTRUMENTO PARA LA APLICACIÓN DESCENTRALIZADA DEL DERECHO EUROPEO DE LA COMPETENCIA. 4. LA RED ESPAÑOLA DE AUTORIDADES DE COMPETENCIA Y SU FUTURO 4.1. La aplicación descentralizada de la competencia en España. 4.2. La REC en un sistema de competencias excluyentes: la necesidad de señalar a la autoridad mejor situada 4.3. El Consejo de Defensa de la Competencia: acto y potencia 5. CONCLUSIONES: HACIA UNA VERDADERA RED ESPAÑOLA DE AUTORIDADES DE COMPETENCIA.
\end{abstract}

\section{LAS REDES Y LA APLICACIÓN DESCENTRALIZADA DEL DERECHO DE LA COMPETENCIA}

No cabe duda de que vivimos en una sociedad de «redes». Todos -o casi todos- formamos parte de una o varias de ellas, ya sean sociales, profesionales, académicas o de cualquier otra índole. El mundo de la competencia no es ajeno a esta realidad y por ello, en el específico campo del Derecho de la Competencia, existen diversos ejemplos de redes, tanto públicas como privadas. 
En el presente trabajo nos vamos a centrar en tres de ellas, que presentan como rasgo común el hecho de estar formadas, total o parcialmente, por autoridades de competencia, lo que las dota de un cierto carácter «institucional», aunque sobre esta idea y sus matizaciones nos referiremos de inmediato. Por otro lado, debemos advertir que emplearemos el término «red» en un sentido amplio, es decir, dando tal calificación tanto a las de contenido virtual (se denominan redes porque conectan instituciones o personas), como aquellas que tiene una estructura organizativa de carácter institucional, de la que surge el «efecto red», que vamos a destacar.

A pesar de este elemento común, la presencia y/o participación de autoridades de competencia, sus diferencias son notables, yendo más allá de la obvia de sus respectivos ámbitos territoriales de actuación. Estas diferencias, lejos de hacerla incompatibles permiten hablar más de complementariedad, sin que sea posible ignorar, no obstante, la existencia de una cierta «rivalidad ${ }^{1}$ entre ellas, fundamentalmente debida al origen de sus recursos, procedentes de los estados que las integran.

Su estudio conjunto nos permitirá no sólo conocerlas mejor, sino apreciar su aludida complementariedad, sin perder de vista el objetivo, -sólo hace unos años inimaginable-, de una futura convergencia ${ }^{2}$ si no institucional, sí al menos, -y ello puede ser más factible y relevante-, en la propia regulación y aplicación global ${ }^{3}$ del Derecho de la Competencia.

Esta interesantísima vertiente de la cuestión no será abordada en esta ocasión, pues excedería los límites del planteamiento general de este número monográfico de la Revista. Nuestra atención se centrará en el análisis de estas redes en tres ámbitos diferentes, el global, representado principalmente por la International Competition Network (ICN), el regional, -en los términos ya apuntados-, centrándonos en el caso de la European Competition Network $\left(\mathrm{ERC}^{4}\right)$ para, en tercer lugar, y a partir de análisis de los anteriores ejemplos,

${ }^{1}$ Esta idea es apuntada con claridad por Kovacic, W. E./Hollman, H., «The International Competition Network: Its Past, Current, and Future Role», Scholary Commons, George Washington University Law School, pp. 274 a 323, en concreto, p. 286. Este excelente trabajo se puede descargar en: http://www.lti.lv/\#! vieslekcijas/cvdu

${ }_{2}$ Resultan especialmente relevantes las reflexiones que acerca de esta cuestión realiza el Profesor R. Whish en una brillante conferencia dictada en 2014, que se puede reproducir en la red: «Prof. Richard WHISH QC (Hon) guest lecture at the Latvian Law Institute, Riga, Latvia», May 30, 2014, disponible en la dirección http://www.lti.lv/\#!vieslekcijas/cvdu

3 Acerca de las particularidades del fenómeno de la globalización en el ámbito de la competencia, vid. GERBER, D. J., Global Competition: Law, Markets and Globalization, Oxford University Press, 2010.

${ }^{4}$ Empleamos para este estudio su denominación en inglés y su correspondiente abreviatura, para evitar la confusión con la Red Española de Autoridades de Competencia, que convencionalmente se denomina como «REC», que son las siglas de la ECN en castellano. 
centrar nuestra reflexión constructivamente crítica, en el caso de la que denominamos Red Española de Autoridades de la Conmpetencia (REAC), cuya consolidación y potencialidad está todavía lejos de ser una realidad, aunque su importancia para una eficiente aplicación descentralizada del Derecho de la Competencia en España resulta incuestionable.

Finalmente debemos señalar que en la medida en que el propósito de este numero de la Revista es profundizar en la «Aplicación descentralizada del Derecho de la Competencia», nuestro examen de dichas redes tendrá esta orientación, teniendo especialmente presente que dos de ellas, la ECN y la REC tiene su origen, precisamente, en dicha aplicación descentralizada, en el ámbito de la Unión Europea y de España, respectivamente 5 .

\section{LA INTERNATIONAL COMPETITION NETWORK (ICN): UNA RED DE COMPETENCIA A ESCALA PLANETARIA}

Lo primero que resulta preciso subrayar es que la ICN no es la única, ni siquiera la más antigua de las redes de competencia de carácter internacional y vocación universal ${ }^{6}$ de las que existen en la actualidad. Sin embargo, debemos anticipar ya que alguno de sus rasgos la hacen especialmente singular y la diferencian de otras redes con las que comparte, al menos en parte, objetivos y ámbito de actuación potencial y cuyas limitaciones, en parte, viene a corregir y superar.

En efecto, junto a la ICN, y nacidas con anterioridad a ella, podemos citar, entre otras, el Competition Law and Policy Committee (CLPC), de la Organización para la Cooperación y Desarrollo Económicos (OCDE) $)^{7}$; la Conferencia de las Naciones Unidas sobre Comercio y Desarrollo (más conocida por sus siglas en inglés, como la $\mathrm{UNCTAD}^{8}$ ), en el marco de las Naciones Unidas y la Organización Mundial de Comercio (OMC). Estos ejemplos, sin

${ }^{5}$ Como tendremos ocasión de señalar, ambas dimensiones quedan claramente delimitadas, en la medida en que la descentralización en España sólo alcanza la de las infracciones sin dimensión comunitaria, pues en nuestro Derecho interno la competencia para aplicar los artículos 101 y 102 TFUE se reserva en exclusiva a la CNMC.

${ }^{6}$ Empleamos el término universal en un doble sentido, pues nos referimos tanto a vocación de agrupar a miembros, sean o no Estados, de todo el planeta, como en el sentido de que su ámbito territorial aunque tenga ciertas limitaciones no se circunscriba a una determinada región o área geográfica (Europa, América,...).

7 También conocida por sus siglas en inglés como la OCDE (Organisation for Economic Co-operation and Development), creada tras la firma en París de la «Convención de la OCDE», el 14 de diciembre de 1960. El texto en español de esta Convención puede consultarse en la web oficial de la organización, en la dirección: http://www.oecd.org/ centrodemexico/laocde/convenciondelaocde.htm

${ }^{8}$ United Nations Conference on Trade and Development. 
embargo, no son ni los únicos, ni siquiera los más antiguos ${ }^{9}$, pero sí perviven en la actualidad y, lo que es más importante, pueden evolucionar o integrarse entre sí o, al menos, cooperar conjuntamente.

La exposición de las características de la ICN se hará, en buena medida, en contraposición a las de las otras tres, y será precisamente lo que la diferencia de aquéllas, lo que explique que la ICN se haya convertido, en muy poco tiempo, en un actor fundamental del panorama de la competencia en el ámbito internacional.

De las redes internacionales «de competencia» que vamos a examinar, es la OCDE la más antigua. Su creación se remonta a 1961 y su marcado carácter «institucional» viene determinado por sus dos características principales: sólo pueden ser miembros «plenos» Estados desarrollados ${ }^{10}$ y son éstos los que proveen de financiación al organismo.

Ambas notas (Estados más desarrollados y financiación de los Estados) determinan, en buena medida, el principal problema de esta organización, pues condicionan tanto sus objetivos como su capacidad real al depender de la financiación de sus miembros que puede verse influida por la coyuntura política de cada momento.

En el ámbito específico de la competencia, las actividades de la OCDE se desarrollan a través del Competition Law and Policy Committee (CLPC), que actúa como foro de debate entre sus miembros en asuntos de competencia y promueve la armonización de normas y criterios entre ellos. Adicionalmente, en el seno de la OCDE se ha creado el Grupo sobre Comercio y Competencia (Group on Trade and Competition) para analizar las relaciones entre ambos ámbitos ${ }^{11}$. Es preciso subrayar, no obstante, que en los últimos tiempos la OCDE está incrementando sus actuaciones dirigidas a Estados no miembros de la organización ${ }^{12}$.

9 Diversos autores dan cuenta de proyectos fallidos de coordinar las políticas de competencia a escala global e incluso con el propósito de elaborar un marco normativo internacional en materia de competencia, iniciados con anterioridad a los que examinamos en este trabajo. A título ilustrativo, THEM Do, K., «The Perspectives of International Cooperation in Competition Law and Policy», en ZweR, $n^{\circ} 3$, 2009, pp. 289 a 314; en concreto, pp. 291 y 292, cita los de la International Trade Organization (ITO) en 1948, el Economic and Social Council of United Nations (ECOSOC), en 1953 o el General Agreement on Tariffs and Trade (GATT), en 1965, que por diversas razones no alcanzaron el éxito. Este interesante y documentado trabajo puede consultarse en: file://C:/Users/jrodriguezm/ Downloads/SSRN-id1554065.pdf

${ }^{10}$ La representación de los Estados miembros de la OCDE tiene un alto carácter de representación que, en algunos casos, como el de España, por ejemplo, tiene rango diplomático.

${ }^{11}$ Cfr. Them Do, K., «The Perspectives..., op. cit., p. 294.

12 Cfr. Them Do, K., «The Perspectives..., op. cit., p. 294. 
A lo largo de sus años de existencia, la OCDE ha adoptado numerosos documentos, sin carácter vinculante (soft law), principalmente recomendaciones, informes ${ }^{13} \mathrm{y}$ catálogos de «buenas prácticas» en materia de cárteles, concentraciones económicas y cooperación ${ }^{14}$. Desde hace algunos años, además, la OCDE promueve un Forum Global de Competencia (Global Forum on Competition o GFC $)^{15}$ que reúne a funcionarios de alto nivel procedentes de las autoridades de competencia de más de noventa países para debatir temas de competencia.

Por su parte, la UNCTAD, fue creada sólo unos años después, concretamente en 1964, en el marco de la Organización de las Naciones Unidas (ONU). A diferencia de la OCDE, los destinatarios de sus actividades no son los países más desarrollados, sino, precisamente, los países menos desarrollados o en vías de desarrollo, lo que hace ambas organizaciones complementarias. Su objetivo principal en sede de competencia y protección de los consumidores es asegurar que los países miembros de la organización disfruten los beneficios de la competencia y de la inversión privada en sectores clave de sus economías y que ello redunde en un incremento del bienestar de los consumidores ${ }^{16}$.

Sin embargo, y al igual que ocurre con la OCDE, el principal problema de la UNCTAD radica en que su financiación procede también de sus miembros, por lo que sus actividades quedan igualmente condicionadas por los recursos que se le transfieran.

Las actividades de la UNCTAD en el ámbito de la competencia se llevan a cabo a través de un programa específico, el «Programa sobre las Políticas de Competencia y Consumo» (Competition and Consumer Policies), que cuenta con un reducido cuadro de personal propio (Competition and Consumer Policy Branch), que coordina la actuación en esta área tanto de un grupo intergubernamental creada a tal efecto, el «Grupo Intergubernamental de Expertos sobre Derecho y Política de la Competencia» (GIE) y de un Grupo ad hoc de Expertos en materia de protección a los consumidores.

La UNCTAD ${ }^{17}$ sirve como foro de debate para los representantes de los Gobiernos que forman parte de la organización acerca de cuestiones relacio-

${ }^{13}$ Una relación de los Informes elaborados por el CLPC puede consultarse en: http:// www.oecd.org/regreform/sectors/reportsbythecompetitioncommittee.htm

${ }^{14}$ Los textos de estas recomendaciones y catálogos de buenas prácticas pueden consultarse en: http://www.oecd.org/competition/recommendations.htm

$15 \mathrm{http}$ //www.oecd.org/competition/globalforum/abouttheglobalforumoncompetition.htm

${ }^{16}$ Cfr. Web oficial UNCTAD, en la dirección: http://unctad.org/en/Pages/DITC/ CompetitionLaw/Competition-Law-and-Policy.aspx

17 Para más detalles acerca de los objetivos y actividades de la UNCTAD es útil consultar la web oficial del organismo, en la dirección: http://unctad.org/en/Pages/About\%20 
nadas con la política de la competencia, principalmente a través de la reunión anual del GIE, pero también desarrolla una importante labor de investigación, análisis y documentación en temas de competencia y consumo y, sobre todo, desarrolla una importante labor proveyendo de asistencia técnica a los países en vía de desarrollo asociados con el fin de ayudarles a seguir las recomendaciones y buenas prácticas identificadas en el marco de sus actividades ${ }^{18}$.

A la hora de resumir sus actividades no debemos olvidar dos logros especialmente relevantes, aunque su grado de eficacia real haya sido relativo. Nos referimos, por una parte, al texto codificado en 1980 bajo el título «Conjunto de Principios y Normas sobre Competencia de las Naciones Unidas» (más conocido como el «UN Set») ${ }^{19}$, que se considera el primer «código internacional de competencia» ${ }^{20}$, y que aunque es obvio, no ha logrado su objetivo principal, su implantación general, es una clara referencia de a dónde se puede llegar, siendo su revisión cada cinco años, en el seno de las UNCTAD ${ }^{21}$, una oportunidad para debatir sobre el proyecto e ir actualizando su contenido.

Junto al «UN Set», la UNCTAD ha aprobado una «Ley-Tipo de Defensa de la Competencia» ${ }^{22}$, a disposición de los países -especialmente en vías de desarrollo- que deseen implantar una legislación de competencia ${ }^{23}$. Este mo-

UNCTAD/A-Brief-History-of-UNCTAD.aspx

${ }^{18}$ Esta labor de asistencia técnica ha sido especialmente intensa con América Latina, a través del programa COMPAL. Vid. a este respecto, http:/www.programacompal.org/

19 El subtítulo de este documento es más expresivo: «Conjunto de principios y normas equitativos convenidos multilateralmente para el control de las prácticas comerciales restrictivas». Este documento puede consultarse en: http://unctad.org/es/docs/tdrbpconf10r2.sp.pdf

${ }^{20}$ Cfr. Themn Do, K., «The Perspectives..., op. cit., p. 292.

${ }^{21}$ Acerca de esta Conferencia y su actividad revisora, vid. United Nations Conference for the Review of the Set, en la web oficial de las UN en la dirección: http://unctad.org/en/ Pages/DITC/CompetitionLaw/United-Nations-Conference-for-the-Review-of-the-Set. aspx

${ }_{22}$ El punto de partida para la elaboración de esta ley-tipo se encuentra en la reunión del Grupo Intergubernamental de Expertos sobre Derecho y Política de la Competencia celebrada los días 7 al 9 de junio de 1999, que fue aceptada por la UNCTAD, que estableció también el carácter revisable del documento, cuya primera versión data del año 2000 (http://unctad.org/en/Docs/tdrbpconf5d7.en.pdf). El texto y sus sucesivas revisiones puede consultarse en: http://unctad.org/en/Pages/DITC/CompetitionLaw/The-Model-Lawon-Competition.aspx

${ }^{23}$ Este modelo no es el único existente pues tanto el Banco Mundial como la OCDE han llevado a cabo iniciativas parecidas. Vid. LEE. C. H., «Model Competition Laws: The World Bank-OECD and UNCTAD Approaches Compared» in C. LEE \& C. MAY-FONG (Eds.), Competition Policy in Asia, 2006. 21-35. Disponible en: http://works.bepress.com/clee/4 
delo, también sometido a continuas revisiones, constituye una pauta de mínimos cuyo alcance práctico es indudable para fomentar la competencia en los países que todavía permanecen al margen de esta regulación.

El tercer ejemplo que vamos a examinar antes de centrarnos en la ICN es el de la OMC. Aunque la OMC encuentra su precedente en los Acuerdos del GATT de 1947, su actual forma y configuración como organización internacional nos lleva a 1994, año en el que tras las largas negociaciones de la llamada «Ronda de Uruguay» del GATT (1986-1994), se firmó su Acta Final, a la que se incorporaron tanto el Acuerdo por el que se establecía la propia OMC (o Acuerdo sobre la OMC), como en diversos anexos, los más de 60 acuerdos alcanzados en el curso de las negociaciones.

Los avances de esta Ronda de negociaciones fueron especialmente importantes, pues además de crear una estructura organizativa con entidad propia, de la que carecía el GATT, se lograron acuerdos en materias como el comercio de mercancías, de servicios, de propiedad intelectual, la solución de diferencias, el mecanismo de examen de las políticas comerciales y se suscribieron numerosos acuerdos plurilaterales ${ }^{24}$.

La OMC se configuró desde un principio como un marco institucional común para el desarrollo de las relaciones comerciales entre sus Miembros en los asuntos relacionados con los acuerdos e instrumentos jurídicos conexos que también se habían aprobado y que resultan vinculantes para los $\operatorname{mismos}^{25}$.

Es preciso reconocer el éxito de la OMC, pues en la actualidad está integrada por 160 países $^{26} \mathrm{y}$ varios de los acuerdos que mejor han funcionado tienen un indudable alcance antitrust, como los relativos al comercio de mercancías (GATT), servicios $\left(\right.$ GATS $^{27}$ ) o los relativos a Subvenciones y Mediadas Compensatorias y a Medidas Antidumping. Lo cierto es que no se llegó, ni entonces, ni es previsible a corto plazo al consenso necesario para aprobar

${ }^{24}$ La relación y el texto de los acuerdos del GATT 1994 se pueden consultar en la web oficial de la OMC en la dirección: https://www.wto.org/spanish/thewto_s/whatis_s/ inbrief_s/inbr03 s.htm

${ }_{25}$ Ártículo II. Ámbito de la OMC

${ }^{26}$ El status de miembro se ha reconocido también a la propia Unión Europea (a este respecto, vid. https://www.wto.org/spanish/thewto_s/countries_s/european_ communities_s.htm), existiendo un amplio número de observadores integrado por Gobiernos que todavía no se han incorporado a la condición de miembros así como por un amplio abanico de organizaciones internacionales intergubernamentales (la lista de Miembros y Observadores se puede consultar también en la web oficial de la OMC en la dirección: Miembros y Observadores, en la dirección https://www.wto.org/spanish/ thewto_s/whatis_s/tif_s/org6_s.htm).

${ }_{27}$ General Agreement on Trade in Services, aprobado en la Ronda Uruguay del GATT. 
acuerdo alguno de contenido propiamente antitrust ${ }^{28}$, pese a ser, sin duda, como ha destacado la Doctrina, la OMC el vehículo más adecuado para hacer frente al difícil reto de un sistema de competencia a escala global ${ }^{29}$.

Es en este contexto donde se crea en 2001 la $\mathrm{ICN}^{30}$, cuya naturaleza y especiales características permiten considerar con fundamento ${ }^{31}$ que puede estar llamada a jugar un papel decisivo en la promoción de la competencia a escala global, a pesar de que sea preciso reconocer, como entre otros afirma Them $\mathrm{Do}^{32}$, que el panorama de la política internacional actual resulta bastante ilusorio creer en la posibilidad de alcanzar un consenso general que permita adoptar un código internacional de competencia que pueda ser aplicado por una autoridad internacional única, pues los Estados miembros no se muestran proclives a limitar su soberanía en una materia tan sensible.

Como ya hemos anticipado implícitamente al aludir a sus predecesoras, la creación de la ICN responde, en buena medida, a las limitaciones advertidas

${ }^{28}$ El tema de la aprobación en el seno de la OMC de un acuerdo global y específico en materia antitrust se suscitó en la Conferencia Intergubernamental de Singapur (1996) de la que surgió la creación de un Grupo de Trabajo destinado a analizar las interrelaciones entre la política comercial y la de competencia, cuyos resultados pasarían posteriormente a la «Agenda de Doha», con motivo de la siguiente conferencia intergubernamental de 2001 en busca de un consenso, que finalmente no fue alcanzado en la ulterior reunión de Cancún (2003), adoptándose finalmente en 2004, en el marco del «July 2004 package» la decisión de 1 de agosto de 2004, del Consejo General de la OMC la decisión de que el debate sobre la política de la competencia «will not form part of the Work Programme set out in that Declaration and therefore no work towards negotiations on any of these issues will take place within the WTO during the Doha Round». Por lo que en la actualidad, el grupo de trabajo que se había creado está actualmente inactivo. El texto de esta Decisión, identificada como «WT/L/579», puede consultarse en: https://www.wto.org/english/ tratop_e/dda_e/draft_text_gc_dg_31july04_e.htm Acerca de esta cuestión, vid. por todos, KovaCic, W. E./HoLlmaN, H., «The International ..., op. cit., pp. 300 y 301.

29 Vid. en este sentido, THem Do, K., («The Perspectives..., op. cit., pp. 290 y 291), o), quien recuerda el estado de la cuestión tras la declaración cerrando, al menos temporalmente, el debate, del Secretario General de la OMC al decidir, el 1 de agosto de 2004, que la cooperación internacional en materia de competencia quedaba excluida del marco de trabajo de la Ronda de Doha.

${ }^{30}$ Como se ha señalado su creación efectiva encontró el momentum preciso a raíz del caso GE/Honeywell y la diferente percepción de esta operación de concentración económica entre la Administración antitrust estadounidense y la Comisión Europea. Acerca de este desencuentro, vid.., entre otros, Donna E. PATterson/CARL Shapiro «Transatlantic Divergence in GE/Honeywell: Causes and Lessons» en Antitrust Magazine, ABA Section of Antitrust Law, Fall, 2011, pp. 18 a 26. Accesible en: http://faculty.haas.berkeley.edu/ shapiro/divergence.pdf

31 Vid. Kovacic, W. E./Hollman, H., «The International..., op. cit., p. 276.

${ }^{32}$ Cfr. Them Do, K., «The Perspectives..., op. cit., p. 291. 
en aquéllas. Como señalan Kovacic y Hollman ${ }^{33}$, el gran objetivo de la ICN es facilitar la convergencia en las grandes cuestiones del Derecho de la Competencia, como son sus aspectos substantivos, procedimentales y administrativos. Con este propósito, la ICN se implica en proyectos destinados a (1) incrementar el conocimiento de los diferentes sistemas individuales de competencia, examinando las diferencias y similitudes entre ellos; (2) identificando y buscando el consenso respecto de las grandes cuestiones del Derecho de la competencia, y (3) apoyando a las diferentes jurisdicciones nacionales para mejorar su nivel técnico.

La singularidad de la ICN frente a las otras redes examinadas obedece a diversos motivos. En primer lugar, no es una red ni de gobiernos ni de de autoridades de competencia, pues aunque participen en ella, no se excluye la presencia tanto de organizaciones internacionales, como académicos o expertos individuales, que también participan en sus actividades, lo que indudablemente enriquece los debates.

En segundo lugar, es una red «prácticamente virtual», pues no es un organismo internacional y carece prácticamente de estructura institucional propiamente dicha, lo que dota de mayor agilidad a la ICN y reduce sus costes y consecuentemente incrementa su independencia real, pues, como tercer nota general, la financiación de la ICN no depende los Estados.

Las actividades de la ICN son dirigidas desde la Directiva de la ICN (Steering Group) y mantiene 5 Grupos de Trabajo activos ${ }^{34}$ : Advocacy, Agency Effectiveness, Cartel, Merger y Unilateral Conduct, que han impulsado diversas recomendaciones y catálogos de buenas prácticas ${ }^{35}$, así como Workshops y una conferencia internacional anual.

\section{LA EUROPEAN COMPETITION NETWORK (ECN) COMO INSTRUMENTO PARA LA APLICACIÓN DESCENTRALIZADA DEL DERECHO EUROPEO DE LA COMPETENCIA}

El origen y la finalidad de la ECN es más claro y concreto, pues se configura como un instrumento al servicio de una eficiente aplicación descentralizada de los artículos 101 y 102 TFUE, que son los que regulan la prohibición de los acuerdos colusorios y el abuso de posición dominante en el marco de la Unión Europea.

Se trata, a diferencia de los ejemplos ya examinados, de una red de competencia de ámbito regional. El interés de su estudio radica no sólo en que es la red en cuyo contexto jurídico nos desenvolvemos como miembros de la

\footnotetext{
33 Vid. Kovacic, W. E./Hollman, H., «The International..., op. cit., p 275.

${ }^{34} \mathrm{http}: / / \mathrm{www}$.internationalcompetitionnetwork.org/working-groups/current.aspx

${ }^{35} \mathrm{Cfr}$. http://www.internationalcompetitionnetwork.org/about/icnguidance.aspx
} 
Unión Europea, sino también en que, como en el caso de la Red Española de Autoridades de Competencia, su creación surge con motivo del proceso de descentralización de la aplicación administrativa del Derecho de la competencia, por lo que su estudio nos ayudará a perfilar cómo debería ser la red española cuya existencia real es muco más limitada que la de la ECN.

La ECN se configura como red en virtud de la aprobación del Reglamento (CE) $n^{\circ} 1 / 2003$, del Consejo de 16 de diciembre de 2002, relativo a la aplicación de las normas sobre competencia previstas en los artículos 81 y 82 [hoy 101 y 102 TFUE] del Tratado ${ }^{36}$ (en adelante, el «Reglamento 1/2003»), junto al que se aprobó también la menos conocida Declaración común del Consejo y de la Comisión sobre el funcionamiento de la Red de Autoridades de Competencia $^{37}$, en la que, entre otros extremos se formulan los principios generales que regirán la red, mientras que los «pormenores» ${ }^{38}$ de su funcionamiento $\mathrm{y}$, en especial, criterios de asignación, se desarrollan a través de un instrumento de soft law: la Comunicación de la Comisión sobre la cooperación en la Red de Autoridades de Competencia («Comunicación sobre la REC», o como ya expusimos para evitar confusiones, «Comunicación ECN») $)^{39}$

Con la aprobación del Reglamento 1/2003, la Comisión renunció al monopolio que hasta entonces venía ejerciendo en la aplicación pública de dichos preceptos; al amparo del conocido como el «Reglamento 17», en vigor desde 1962, que es al que reemplaza el Reglamento $1 / 2003^{40}$.

El Reglamento 1/2003 supuso además el abandono del sistema de notificación previa de los acuerdos que inicialmente prohibidos por el artículo 101.1 TFUE, podían ampararse en la excepción que se recoge en el apartado $3^{\circ}$ del mismo precepto ${ }^{41}$, en favor de un sistema, denominado de «excepción

${ }^{36}$ DO L 1, de 4.1.2003, p. 1.

37 Esta Declaración común se puede descargar en http:/ec.europa.eu/competition/ ecn/joint_statement_es.pdf

${ }_{38}$ Cfr. Declaración común..., cit. punto 4.

39 DO C 101, de 27.4.2004, p. 43. Como recuerdan ORTIZ BlANCO, Luis y LAMADRID DE PABLO, Alfonso («EU Competition Law Enforcement: Elements for a discussion on effectiveness and uniformity«, en International Antitrust Law \& Policy: Fordham Competition Law 2011, Chapter 4, 2012, pp. 45 a 104, en concreto, 100, nota 222), este documento no sólo vincula a la Comisión, sino también a los Estados miembros que suscribieron, como se recoge en la propia Comunicación (nota 4), la Declaración común del Consejo y de la Comisión sobre el funcionamiento de la Red de Autoridades de Competencia, disponible en el registro del Consejo, en http://register.consilium.eu.int (documento no $15435 / 02$ ADD 1).

${ }^{40}$ Reglamento (CEE) $n^{\circ} 17$ del Consejo: primer reglamento de aplicación de los artículos 85 y 86 del Tratado (DO 13 de 21.02.1962).

${ }^{41}$ El sistema de notificación previa obligatoria pervive, en mayor o menor grado en otros campos del Derecho Europeo de la Competencia como el de las concentraciones económicas y las ayudas de Estado. 
legal», en el que son los propios operadores económicos quienes deben evaluar (autoevaluación) sus prácticas a la luz de todo el artículo 101 TFUE.

La conveniencia de crear una red de autoridades de competencia para facilitar la aplicación del nuevo sistema es planteada por el propio Reglamento $1 / 2003$, reconociéndose además la necesidad de crear mecanismos de información y de consulta ${ }^{42}$.

En la medida en que el Reglamento 1/2003 se construye bajo la premisa de que las competencias de la Comisión y las Autoridades nacionales de la Competencia (ANC) son concurrentes (y no repartidas de forma excluyente) ${ }^{43}$, sus respectivas funciones, así como en su caso la de los órganos judiciales nacionales que resuelven litigios entre particulares, resultan necesariamente complementarias $^{44}$.

De este modo, y aunque se afirme que la ECN se configura como un «foro de discusión y cooperación», lo es «para la aplicación y el control del cumplimiento de la política comunitaria de competencia», por lo que con su creación se pretende proporcionar un marco estable y definido para la cooperación de las autoridades europeas de competencia en la aplicación de los artículos 101 y 102 TFUE, pero que, al mismo tiempo, sirva de base para «crear y mantener una cultura común de competencia en Europa. $\rangle^{45}$

El papel de la ECN resulta vital y debe su éxito, en no poca medida, a la flexibilidad con la que el principio básico de las competencias concurrentes ha sido diseñado y aplicado ${ }^{46}$.

${ }^{42}$ Cfr. Punto 15 de la parte expositiva del Reglamento 1/2003. Esta «sugerencia» se completa con la que se exponen en los tres puntos siguientes de la misma parte dispositiva del Reglamento 1/2003 (Punto 16 a 18), que ofrecen una pautas claras para la interpretación de las previsiones que el propio reglamento incorpora para hacer efectiva la aplicación descentralizada de los artículos 101 y 102 TFUE.

${ }^{43}$ La Comunicación ECN habla claramente de «división del trabajo» (epígrafe 2), afirmando que un sistema de competencias concurrentes se basa en que «todas las autoridades de competencia tienen potestad para aplicar los artículos 81 y 82 del Tratado y deben buscar un reparto eficaz de los asuntos cuando se considere que una investigación es necesaria. Al mismo tiempo, cada miembro de la Red es totalmente libre para decidir si es o no oportuno investigar un asunto.» (punto 5, párr. primero).

${ }_{44}$ Así se recoge en el punto 1 de la Comunicación de la Comisión sobre la tramitación de denuncias por parte de la Comisión al amparo de los artículos 81 y 82 del Tratado CE (DO C 101, de 7.4.2004, p. 65).

${ }^{45}$ Cfr. Comunicación ICN, punto 1 .

46 No se trata, sin embargo, del criterio general en el ámbito de la competencia pues, por ejemplo, en materia de Ayudas de Estado, donde la Comisión tiene competencias exclusivas o en sede de concentraciones la Comisión o las ANC «tendrá(n) competencia exclusiva para adoptar las decisiones que correspondan, conforme al Reglamento (CE) $\mathrm{n}^{\circ}$ 139/2004 del Consejo, de 20 de enero de 2004, sobre el control de las concentraciones entre empresas (DO L 24, de 29.01.2004, p. 1), cuando se aprecie que tienen «dimensión 
La asignación de casos entre la Comisión Europea y/o una o varias ANC no responde a criterios rígidos, sino que es aplicado de manera flexible, lo que contrasta acentuadamente con lo que ocurre en el ámbito del Derecho español de la competencia, tal y como ha sido interpretado por nuestro Tribunal Constitucional, se asienta en un sistema de «competencias excluyentes», que se aplica atendiendo a criterios en exceso formalistas y, a la postre, mucho menos claros y eficientes ${ }^{47}$.

Como ya anticipamos, los criterios de asignación de casos en el marco de la ECN están recogidos en la Comunicación $\mathrm{ECN}^{48}$. En primer lugar, y con carácter previo al proceso de asignación, debe resolverse si la conducta cuestionada afecta o puede afectar al mercado intracomunitario o sus efectos (actuales o potenciales) se limitan únicamente a todo o parte de un Estado miembro (afectación al comercio entre Estados miembros) ${ }^{49}$.

De apreciarse la afectación del mercado intracomunitario, los asuntos podrán ser tramitados: a) por una única autoridad nacional de competencia, posiblemente con la ayuda de las de otros Estados miembros; b) por varias autoridades nacionales de competencia actuando de forma concurrente; o c) por la propia Comisión ${ }^{50}$.

comunitaria» o solamente nacional, lo que se hace en función de parámetros concretos y fijos previstos en la propia norma. En materia de concentraciones resulta de interés la STPI sentencia de 14 de julio de 2006, Endesa/Comisión, asunto T-417/05, Rec. 2006 p. II-2533, a propósito de la frustrada operación Gas Natural/ENDESA.

${ }^{47}$ En el punto 5 de la Comunicación de la Comisión sobre la cooperación en la Red de Autoridades de Competencia (DO C 101, de 27.4.2004, p. 43) se señala «5. El Reglamento [1/2003] del Consejo se basa en un sistema de competencias concurrentes en el que todas las autoridades de competencia tienen potestad para aplicar los artículos 81 y 82 del Tratado y deben buscar un reparto eficaz de los asuntos cuando se considere que una investigación es necesaria. [...]»

${ }_{48}$ DO C 101, de 27.4.2004, p. 43.

49 Artículos 101.1 y 102 TFUE. Esta fundamental cuestión es abordada por la Comunicación de la Comisión - Directrices relativas al concepto de efecto sobre el comercio contenido en los artículos 81 y 82 del Tratado [hoy artículos 101 y 103 TFUE] (DO C 101 de 27.4.2004, p. 81). Por lo general, como recuerda GIPINI, en los casos de aplicación del artículo 101 TFUE sólo será precisa la delimitación del mercado relevante cuando sea el único medio que permita establecer la afectación de la competencia o del mercado entre Estados miembros [GIPPINI FouRnIER, E.: Community...op. cit., p. 72. Sin perjuicio, claro está, de que sea preciso para determinar la cuota de mercad a efectos de la aplicación de alguna exención por categorías como las previstas en el Reglamento (UE) $\mathrm{n}^{\mathrm{o}} 330 / 2010$, de la Comisión, de 20 de abril de 2010, relativo a la aplicación del artículo 101, apartado 3, del Tratado de Funcionamiento de la Unión Europea a determinadas categorías de acuerdos verticales y prácticas concertadas (DO L L 102, de 23.04.2010, p. 1) en relación con las Directrices relativas a las restricciones verticales (DO C 130, de 19.5.2010, p. 1).

${ }^{50}$ Cfr. Comunicación ECN, punto 5. 
Como reconoce la Comunicación ${ }^{51}$, en la mayoría de los casos, será la autoridad que recibe una denuncia o incoe de oficio un procedimiento la que seguirá siendo responsable del mismo. No obstante, se prevé la posibilidad de un «segundo reparto» de un asunto, que solamente se plantearía al principio del procedimiento cuando la autoridad a la que le correspondería inicialmente considerase que «no está bien situada para actuar o cuando otras autoridades se consideran también competentes $\left(. .{ }^{52}\right) »$. Es decir, cuando la ANC no se considere competente o, de algún modo quiera rehusar la atribución o cuando exista un «conflicto de atribución» entre varias $\mathrm{ANC}$, que haya que resolver, sin excluir que puedan ser varias de forma concurrente.

Es en este contexto, cuando la ANC que prima facie se consideraría competente no lo estime así, o sean varias las que creen estarlo al mismo tiempo, cuando cobra especial significado la existencia de la ECN.

El debate se suscita así en torno a la expresión, nada ambigua y enormemente flexible ${ }^{53}$, de la Autoridad «mejor situada», si bien esta expresión se reserva, en realidad, para cuando la atribución la pretenda la propia Comisión $^{54}$.

La Comunicación ECN utiliza principalmente la expresión de Autoridad «bien situada», considerando como tal a la que reúna la totalidad de los siguientes requisitos:

${ }^{51}$ Cfr. Punto 6 Comunicación ECN.

${ }^{52}$ En este paréntesis, el punto 6 de la Comunicación ECN se remite a los puntos 8 a 15 de la misma.

${ }_{53}$ Como recuerda FOLGUERA, el Reglamento 1/2003 no establece normas que regulen con carácter imperativo el reparto de asuntos entre la Comisión y las Autoridades nacionales de la competencia, sino que opta porque éste se decida de forma consensuda y flexible en el seno de la propia REC (ECN) (Folguera CRESPO, J.: «El concepto de autoridad bien situada y la cooperación en el seno de la red de autoridades de competencia», en MARTínEZ Lage, S./Petitbó, J. A. (Dirs.): La Modernización del Derecho de la Competencia en España y la Unión Europea, Fundación Rafael del Pino, Madrid, 2005, pp. 209 a 226; en concreto, p. 214. Respecto de los casos de cártel en que se apreció afectación del mercado intracomunitario examinados por la $\mathrm{CNC}$ desde su creación y en los que se consideró que ésta era la Autoridad mejor situada, vid. CORTI VARELA, J.: «Tipología de cárteles un estudio de los 20 casos resueltos por la CNC y la CNMC», en el Capítulo 3 de la presente obra.

${ }^{54}$ Comunicación ECN, punto 8 in fine: «9. Estos criterios indican que debe existir un vínculo material entre la infracción y el territorio de un Estado miembro para que se considere bien situada a la autoridad de competencia de dicho Estado. Puede esperarse que, en la mayoría de los casos, las autoridades de los Estados miembros donde la competencia se ve afectada apreciablemente por una infracción estarán bien situadas, siempre que puedan poner fin efectivamente a la infracción, a través de acciones individuales o concurrentes, a menos que la Comisión esté mejor situada (véanse los puntos 14 y 15). El subrayado es nuestro. 
1. que el acuerdo o práctica tenga efectos reales o previsibles, directos y sustanciales, sobre la competencia en su territorio, se ejecute en su territorio o proceda del mismo;

2. que la autoridad pueda poner efectivamente fin a toda la infracción, es decir, pueda ordenar el cese de la misma de modo que se ponga fin a la infracción y pueda, en su caso, sancionarla adecuadamente;

3. que pueda reunir, posiblemente con la ayuda de otras autoridades, las pruebas requeridas para probar la infracción $\gg{ }^{55}$.

Pero tal condición puede recaer en la propia Comisión, que sería en este caso, siguiendo la terminología de la propia Comunicación ECN, la «mejor situada». Así, la Comisión se considera «particularmente bien situada» (es la «mejor situada»):

1. Cuando «uno o varios acuerdos o prácticas, incluidas las redes de acuerdos o prácticas similares, tienen efectos sobre la competencia en más de tres Estados miembros (mercados transfronterizos que cubren más de tres Estados miembros o varios mercados nacionales) ${ }^{56}$.

2. Cuando el asunto «está estrechamente ligado a otras disposiciones comunitarias cuya aplicación esté reservada a su competencia exclusiva [la de la Comisión] o cuya aplicación por la Comisión redunde en una mayor eficacia, así como en los casos en que el interés comunitario requiera la adopción de una decisión de la Comisión para, a raíz de la

${ }^{55}$ Cuando se aprecie la posible afectación del mercado intracomunitario en relación con conductas que puedan infringirlos artículos 101 y/o 102 TFUE, se deberá dar traslado del caso a la REC en cuyo seno se producen los contactos informales entre la Comisión y las ANC que resultarán en la asignación del caso. No obstante, el carácter confidencial de estos contactos, como en general de los diversos instrumentos de coordinación (tanto los previstos en el Reglamento $1 / 2002$, como en la Ley $1 / 2002$ ), dificulta conocer cuándo y cómo se han producido, aunque se puede deducir su existencia cuando la conducta se haya materializado en más de un Estado miembros. Esta falta de transparencia ha sido apuntada, respecto de los instrumentos de coordinación del Reglamento 1/2003, por Ortiz Blanco, Luis y Lamadrid de Pablo, Alfonso («EU Competition..., op. cit., pp. 97 y 98, con sus correspondientes notas), quienes se hacen eco de la opinión de los interesados en los expedientes que apuntan, frente a la complacencia de la Comisión, que dicha laguna provoca que sus opiniones al respecto sean de carácter básicamente «abstracto» y como en el concreto tema de la asignación de casos, sería más conveniente un sistema que determinara con mayor certidumbre la autoridad competente para conocer del caso. Debemos señalar en este punto que si bien compartimos la primera afirmación, manifestamos nuestras dudas acerca del segundo aspecto, pues la flexibilidad del sistema es una de sus virtudes y la posibilidad de que la Comisión se asigne aquellos casos que considere de interés trata, precisamente, de mantener cierto equilibrio.

${ }^{56}$ Cfr. Punto 14 de la Comunicación ECN. La propia Comunicación ECN, dado su carácter de soft law, se permite dar varios ejemplos de esta situación. 
aparición de un problema de competencia nuevo, desarrollar la política comunitaria de competencia o para velar por su observancia efectiva.» ${ }^{57}$

Este sistema implica, en consecuencia, que la competencia para conocer del posible ilícito antitrust corresponderá, en principio, a la Autoridad o Autoridades mejor situadas; si bien la norma reserva a favor de la Comisión, de forma más o menos pautada, la posibilidad de avocar para sí el asunto.

Así se establece en el en el artículo 11.6 del Reglamento $1 / 2003^{58}$, en el que, tras formular el principio general de que la Comisión y las ANC «aplicarán las normas de competencia comunitarias en estrecha colaboración..» ${ }^{59}$

$\mathrm{Y}$ al exponer los medios de información recíproca que se establecen, afirma que la incoación de un procedimiento por parte de la Comisión con vistas a la adopción de una decisión priva a las ANC de su competencia para aplicar los artículos 101 y 102 TFUE; si bien, si una ANC estuviera actuando ya en un asunto, la Comisión «únicamente incoaría el procedimiento tras consultar con la autoridad nacional de competencia. $\rangle^{60}$

Al mismo tiempo, la ECN ha aprobado diversos documentos de interés, como el ECN Model Leniency Programme, cuya primera versión data de septiembre de $2006^{61}$, así como diversas Recomendaciones ${ }^{62}$, en temas como los poderes de investigación, procedimientos para recabar pruebas en formato digital, entre otras.

Se trata, en suma, de un sistema que ha venido funcionando de manera satisfactoria, al permitir a la Comisión centrarse en los casos más complejos e importantes, por lo general de dimensión internacional (Microsoft, Intel, Google, entre otros), sin que otras prácticas (sean acuerdos o abusos) hayan quedado sin persecución, al intensificarse la labor investigadora y represora de las ANC, ejerciendo el poder que se les ha reconocido desde el 1 de mayo de $2004^{63}$.

${ }^{57}$ Cfr. Punto 15 de la Comunicación ECN.

${ }^{58}$ Bajo el expresivo epígrafe de «Cooperación entre la Comisión y las autoridades de competencia de los Estados miembros»), del que se infiere que la Comisión asume el expediente cuando sus peculiares características lo hagan aconsejable.

59 Artículo 11.1 Reglamento $1 / 2003$.

${ }^{60} \mathrm{La}$ facultad de la Comisión para avocar un caso persiste hasta el momento en que la ANC haya dictado su resolución definitiva; si bien, es requisito ineludible que la Comisión haya procedido a su inacción.

${ }^{61}$ La última versión data de noviembre de 2012. Texto disponible en: http://ec.europa.eu/competition/ecn/mlp_revised_2012_en.pdf

${ }^{62}$ Las Recomendaciones de la $\bar{E} C N$ pueden descargarse en: http://ec.europa.eu/competition/ecn/documents.html

${ }^{63}$ Un examen exhaustivo de los primeros años de funcionamiento de la ECN puede verse en LóPEZ GÁLVEZ, I., «La red española de autoridades ECN. Dos años de cooperación en la aplicación de las normas de competencia», $A d C, \mathrm{n}^{\circ} 1,2006$, pp. 39 a 58.

Idioma: español 


\section{LA RED ESPAÑOLA DE AUTORIDADES DE COMPETENCIA Y SU FUTURO}

La Red Española de Autoridades de Competencia, como veremos en breve, parte de una situación claramente diferente $\mathrm{y}$, a nuestro juicio, mucho mejor, que la que se prevé en el ámbito interno español, donde el principio de descentralización se combina acertadamente con el de eficacia, en beneficio del interés general del correcto y buen funcionamiento del sistema.

\subsection{La aplicación descentralizada de la competencia en España}

La aplicación descentralizada del Derecho español de la competencia se suscitó ya durante la tramitación de la Ley 16/1989, de 17 de julio, de Defensa de la Competencia, primera ley de competencia adoptada tras la aprobación de la Constitución de 1978. Sin embargo, dicha competencia no fue reconocida entonces. Hubo que esperar a que diez años después, el Tribunal Constitucional, en su STC 208/1999, declarara la inconstitucionalidad de determinados aspectos de la Ley 16/1989 ${ }^{64}$, afirmando que las Comunidades Autónomas disponían de competencia ejecutiva para aplicar, bajo ciertos parámetros, la legislación nacional de competencia.

En este contexto, similar, que no igual al de la Unión Europea, de la aplicación descentralizada de la normativa nacional de competencia, se produce la aprobación de la Ley 1/2002, de 21 de febrero, de Coordinación de competencias del Estado y las Comunidades Autónomas en materia de defensa de la competencia (en adelante Ley 1/2002), que siguiendo las indicaciones del $\mathrm{TC}$ respecto al desarrollo ulterior por el legislador estatal de los criterios de

${ }^{64}$ El proceso arranca con la controvertida STC n ${ }^{0}$ 208/1999, de 11 de noviembre [Publicada en el BOE de 16 de diciembre de 1999, $\mathrm{n}^{\circ} 300$ (suplemento) y, su rectificación posterior, en el BOE de 14 de abril de 2000, n 90 (suplemento)]. El TC declaró la inconstitucionalidad de la cláusula «en todo o en parte del mercado nacional», contenida en la versión originaria de la ya derogada Ley 16/1989, de 17 de julio, de Defensa de la Competencia, por desconocer las competencias ejecutivas de las Comunidaddes Autónomas con competencia en materia de «comercio interior». La descentralización de la aplicación del derecho de la Competencia en España es un tema al que hemos dedicado especial atención. A este respecto, vid. Rodríguez Miguez, J. A.: La reforma del sistema español de defensa de la competencia: La descentralización administrativa de la aplicación del Derecho de la competencia en España, Documento de Trabajo, Serie Política de la Competencia, No 22 , CEU Ediciones, 2006. http://hdl.handle.net/10637/3057, «Defensa de la Competencia y Comunidades Autónomas», en Beneyto, J. M. y Maillo, J. (eds): La nueva Ley de Defensa de la Competencia. Análisis y comentarios, Bosch, Barcelona, 2009, pp. 61 a 107 y más recientemente, «Descentralización y eficiencia en la protección de los consumidores y la competencia: el caso español», Revista de Derecho de la Competencia, CEDEC, vol. 10, no 14, Pontificia Universidad Javeriana de Bogotá, Bogota, 2014, pp. 207 a 233. 
asignación, estableció las «reglas de juego» en las que dicha aplicación descentralizada debía desenvolverse. La Ley 1/2002 optó frente al modelo claramente definido del Reglamento 1/2003 y la ECN, sobre la base de competencias concurrentes, por el más rígido de las competencias excluyentes, impulsando, al mismo tiempo, instrumentos para llevar a cabo la imprescindible coordinación que la aplicación descentralizada exige.

Como ya podemos adelantar, a nuestro juicio, el diseño institucional resulta mucho más rígido e ineficiente, propiciando conflicto de atribución, superados en la experiencia española con importantes dosis de lealtad institucional, pero que no ocultan el hecho principal de que un sistema de competencias excluyentes permite a las partes impugnar la asignación efectuada, incluso aunque no hubiera existido conflicto sino consenso entre las administraciones potencialmente competentes, lo que resta eficacia al sistema.

En el sistema de aplicación descentralizada de las normas de competencia españolas no se puede hablar, en puridad, de una verdadera «red de competencia», comparable a la ECN, sino de mecanismos de coordinación y asignación de casos, diseñados en la citada Ley $1 / 2002$, al que se ha añadido a posteriori, una red interna, virtual en sentido propio (electrónica), para tal propósito: la denominada «Red de Cooperación de los Órganos Competentes en materia de competencia (REC)».

De este modo, la REC es un simple recurso informático, articulada en el marco de la plataforma de la administración electrónica de la Administración General del Estado (AGE), gestionado por el Centro de Recursos de Comunicación e Información para Administraciones, Empresas y Ciudadanos $(\mathrm{CIRCABC})^{65}$.

La REC es, en definitiva, como se recoge en un documento de la propia $\mathrm{CNC}^{66}$, «un espacio común de intercambio de información que permite la comunicación y coordinación fluida y permanente entre todas las autoridades de competencia de las distintas Administraciones (Estatal y autonómica)».

Para la actualización de este «espacio común de intercambio de información» se creó y está en funcionamiento desde $2008^{67}$ un Grupo de Trabajo, de los creados en el seno del Consejo de Defensa de la Competencia.

${ }^{65}$ La administración electrónica de la AGE se apoya en Portal de la Administración Electrónica en la dirección http://administracionelectronica.gob.es/ gestionada técnicamente por el CIRCABC.

${ }^{66} \mathrm{CNC}$, Aplicación de la Ley 1/2002, de 21 de febrero, de coordinación de las competencias del Estado y las Comunidades Autónomas en materia de defensa de la Competencia 2008-2012, p. 41. http://www.cncompetencia.es/Portals/0/PDFs/Docs/CNC-infor$\mathrm{me} \% 20$ autonom\%C3\%ADas5_indexado.pdf

${ }^{67}$ En la reunión del Consejo de Defensa de la Competencia, de 19 de dicembre de 2008 se creó el «Grupo de actualización y mantenimiento de la Red de Cooperación de los 
Es, precisamente este organismo, el Consejo de Defensa de la Competencia, el único en sentido estricto (formalmente «no virtual»), que reúne a todas las autoridades españolas de competencia (la actual CNMC y las Comunidades Autónomas), en el que se puede identificar, de forma muy desdibujada lo que el Libro Blanco, que precedió la modélica aprobación de la Ley 15/2007, de 3 de julio, de Defensa de la Competencia, denominó en su día, enfáticamente, el Sistema Español de Defensa de la Competencia ${ }^{68}$.

Vamos a hacer un breve examen crítico y constructivo tanto del sistema de competencia excluyente, como del funcionamiento del Consejo de Defensa de la Competencia.

\subsection{La REC en un sistema de competencias excluyentes: la necesidad de señalar a la autoridad mejor situada}

Frente al modelo de «competencias compartidas» del Reglamento $1 / 2003$, en el que la asignación de casos pivota sobre la base de la autoridad «mejor situada», que no excluye ni la atribución compartida ni la posible avocación por la Comisión Europea, la Ley 1/2002 consagra un sistema de «competencias excluyentes», que no fue alterado tras la aprobación de la vigente LDC, ni tras la creación de la CNMC, desaprovechándose en ambas ocasiones la oportunidad de incorporar su texto a la nueva norma y de flexibilizar el rígido sistema de asignación de casos previsto en aquélla ${ }^{69}$.

En consecuencia y desde una perspectiva práctica, el principio de «competencias excluyentes» veta, por una parte, la posibilidad de que las Autoridades Autonómicas puedan conocer de la aplicación de los artículos $101 \mathrm{y}$ 102 TFUE, que se reserva en exclusiva a la CNMC y, por otro, delimita a partir de unos puntos de conexión ${ }^{70}$, el reparto de competencias entre la

Órganos Competentes en materia de competencia». CNC, Aplicación de la Ley 1/2002..., op. cit. p. 41.

${ }^{68}$ Cfr. Libro Blanco para la Reforma del Sistema Español de Defensa de la Competencia, Ministerio de Economía y Hacienda, 20 de enero de 2005. Disponible en: http:// www.cncompetencia.es/tabid/76/Default.aspx?EntryId=11366\&Command=Core Download\&Method=attachment

${ }^{69}$ No obstante debe reconocerse que la LDC corrigió uno de los rasgos más evidentes de la Ley $1 / 2002$ que era la existencia de notables asimetrías entre las exigencias impuestas al, por aquel entonces SDC, y las requeridas a los órganos autonómicos en materia de competencia. A través de la reforma del Artículo 5.Dos, letra b), de la Ley 1/2002, introducida por la LDC, estos desequilibrios son corregidos de forma sencilla, superando el absurdo de que las Comunidades Autónomas hubieran de mandar copia de todas las denuncias y el SDC únicamente nota sucinta.

${ }^{70} \mathrm{La}$ CNC primero y hoy la CNMC han publicado los resultados de la aplicación de estos puntos de conexión en sucesivos informes: CNC: Aplicación de la Ley 1/2002, de 21 
CNMC y los órganos autonómicos de defensa de la competencia allí donde están constituidos, respecto de los casos «nacionales» (sin incidencia comunitaria $)^{71}$.

Esta atribución exclusiva a la CNMC de los casos de aplicación de los artículos 101 y 102 TFUE se mantuvo tanto con la aprobación de la LDC como tras la creación de la CNMC en la Ley $3 / 2013$, de 4 de junio, de creación de la Comisión Nacional de los Mercados y la Competencia. Únicamente, y como ya se preveía en la LDC, la hoy Dirección de Competencia de la CNMC podrá recabar un informe del órgano autonómico de competencia en cuyo ámbito territorial la conducta incida de forma «significativa» $\rangle^{72}$.

La exclusividad se transforma en un reparto excluyente de competencias entre la CNMC y las autoridades autonómicas de competencia cuando la conducta prohibida carece de incidencia europea. En estos casos el sistema que consagra la Ley 1/2002 exige determinar la autoridad competente en función de que sus efectos se circunscriban exclusivamente al ámbito territorial de una Comunidad Autónoma con autoridad de competencia propia o, afecte a más de una Comunidad Autónoma o al conjunto del territorio nacional.

En estos casos, y con escaso margen de maniobra, la Ley $1 / 2002^{73}$ determina el órgano competente para instruir y resolver, con la posible apelación en caso de disenso a la Junta Consultiva en materia de conflictos, cuyo pronunciamiento puede ser recurrido al propio Tribunal Constitucional ${ }^{74}$.

de febrero, de coordinación de las competencias del Estado y las Comunidades Autónomas en materia de defensa de la Competencia 2008-2012, que incorpora los datos de la edición anterior que recogía el período 2008-2010 y CNMC: Informe sobre la aplicación de los mecanismos de asignación de expedientes CNMC/CCAA y novedades, correspondiente a 2013. Estos informes se pueden consultar en: www.cnmc.es/

${ }^{71} \mathrm{La}$ afectación del mercado intracomunitario no siempre resulta pacífica, especialmente cuando la conducta se puede centrar en una parte de un Estado Miembro. Tal es el caso, por ejemplo, de la Resolución 6 de Octubre de 2011, en el Expte. S/0167/09 Productores de Uva y Vinos de Jerez, en el que se cuestionó que el asunto debiera asignarse a la CNC, en detrimento de la Agencia de Defensa de la Competencia de Andalucía, al cuestionarse la afectación inracomunitaria y circunscribirse a una única provincia andaluza. Vid. FD n ${ }^{\circ} 2^{\circ}$. La afectación del mercado intracomunitario y, por ello, la asignación a la $\mathrm{CNC}$ y no a la Autoridad andaluza fue respaldada por la Audiencia Nacional en su sentencia de 15 de octubre de 2012, $n^{\circ}$ de recurso 608/2011, FD $3^{\circ}$.

72 Conforme a lo dispuesto en el artículo 5. Cuarto, de la Ley 1/2002 y del artículo 33.2 del Real Decreto 261/2008, de 22 de febrero, por el que se aprueba el Reglamento de Defensa de la Competencia (RLDC).

${ }^{73}$ Esta cuestión es tratada exhaustivamente por GUILLÉN CARAMES; J.: «La descentralización..., op. ci., en concreto, pp. 824 a 854.

74 Vid. STC Sentencia 71/2012, de 16 de abril de 2012. Conflicto positivo de competencia 7601-2007. Planteado por el Consejo de Gobierno de la Comunidad de Madrid frente al 


\subsection{El Consejo de Defensa de la Competencia: acto y potencia}

El Consejo de Defensa de la Competencia (CDC) es creado por la Ley $1 / 2002$, regulándolo en el artículo que dedica a los «Mecanismos de coordinación $\rangle^{75}$.

$\mathrm{Al} \mathrm{CDC}$ se le atribuye la condición de «órgano de colaboración, coordinación e información recíproca entre el Estado y las Comunidades Autónomas para promover la aplicación uniforme de la legislación de competencia».

A pesar de que una lectura rápida y superficial de esta previsión podría inducirnos a pensar que el CDC es un órgano equivalente a la ECN o incluso que tendría una formalidad mayor incluso que aquélla, un examen más detallado nos libra del error.

Ello es así, en buena medida, porque la ECN y el CDC se enmarcan en contextos diferentes y en una también diferente comprensión del principio de descentralización administrativa.

La aprobación de la nueva LDC, a pesar de no integrar el texto de la Ley $1 / 2002$, -lo que como ya hemos señalado reiteradamente hubiera sido lo más adecuado $^{76}$-, modifica las funciones del $\mathrm{CDC}^{77}$, al que se apela en diversas ocasiones a lo largo de su articulado.

De acuerdo con la nueva redacción dada al Artículo 5.Uno.3:

«3. El Consejo de Defensa de la Competencia, como órgano de participación y colaboración entre la Administración General del Estado y las Comunidades Autónomas, asumirá las siguientes funciones:

a) Realizar el seguimiento periódico de la política de defensa de la competencia por parte de las distintas Administraciones públicas.

b) Promover el intercambio de información y la realización y publicación de estudios en los que se pongan de manifiesto los criterios seguidos

acuerdo del Pleno del Tribunal de Defensa de la Competencia de 31 de mayo de 2007 por el que se mantiene la competencia de dicho órgano sobre el expediente núm. 627-2007, Estación sur de autobuses de Madrid. Competencia estatal para resolver el expediente sancionador al producirse la hipotética desventaja competitiva en la explotación de una línea de transporte internacional, de modo que la práctica restrictiva tendría incidencia supra-autonómica (STC 208/1999). Con voto particular (BOE núm. 117, de 16 de mayo de 2012).

${ }^{75}$ Cfr. Artículo 5 Ley 1/2002.

76 Vid. Rodríguez Miguez, J. A.: La reforma del sistema español de defensa de la competencia: La descentralización administrativa de la aplicación del Derecho de la competencia en España, Documento de Trabajo, Serie Política de la Competencia, № 22, CEU Ediciones, 2006, pp. 13 a 17 y «Defensa de la Competencia y Comunidades Autónomas», en Beneyto, J. M. y MaILlo, J. (eds): La nueva Ley de Defensa de la Competencia. Análisis y comentarios, Bosch, Barcelona, 2009, pp. 61 a 107; en concreto, p. 72.

${ }^{77}$ La Disposición Adicional Décima, que se incorpora ex novo al texto del PLDC durante su paso por el Congreso de los Diputados. En el Senado se hizo una pequeña adición para mejorar su redacción. 
por las distintas Administraciones en aplicación de la normativa de defensa de la competencia y, en su caso, la necesidad de hacer que éstos sean uniformes.

c) Informar sobre los proyectos de disposiciones de carácter general que afecten a las materias de la defensa de la competencia en las que las Comunidades Autónomas tienen competencias de ejecución.

d) Elaborar directrices sobre la interpretación del apartado 2 del artículo 1 de la presente Ley.»

El único cambio introducido, -y no es nada irrelevante-, afecta a la letra d), que se incorpora al texto del precepto, que sólo recogía las tres funciones anteriores. A partir de ahora descansará sobre el Consejo de Defensa de la Competencia de elaborar directrices sobre la interpretación de los casos en que «ex lege» se considere que existe afectación supra-autonómica y, por lo tanto, la competencia es de la Administración General del Estado. Este mecanismo permitirá matizar notablemente los conceptos recogidos en dicho precepto, que serán interpretados por un órgano en el que las Comunidades Autónomas están también representadas.

No debemos pasar por alto que el CDC ha alumbrado diversos grupos de trabajo (obre criterios de asignación, el funcionamiento de la REC y promoción de la competencia); cuyos frutos son, sin duda, interesantes, pero puede sacarse más partido a la estructura ya existente.

Por su parte, las Autoridades Autonómicas de la Competencia han venido impulsando la celebración anual de unas Jornadas Nacionales de Defensa de la Competencia, asumidas en años sucesivos por una de las autoridades autonómicas, con la colaboración de las demás, sobre temas de actualidad en materia de competencia ${ }^{78}$.

Además de estos supuestos, la propia LDC requiere el concurso del Consejo de Defensa de la Competencia en, al menos, tres ocasiones más:

1. ${ }^{\circ}$ El Artículo 1.5 LDC establece que «[...] el Gobierno podrá declarar mediante Real Decreto la aplicación del apartado 3 del presente artículo a determinadas categorias de conductas, previo informe del Consejo de Defensa de la Competencia y de la Comisión Nacional [de los Mercados y] de la Competencia». Se trata de las conocidas como exenciones por categorías, que al revestir la forma de real de decreto podrían ya considerarse subsumidas en la función c) aludida en la enumeración anterior.

${ }^{78}$ La ultima edición, correspondiente a 2014, se desarrolló en Valencia, siendo organizada por la Comisión de Defensa de la Competencia de la Generalitat Valenciana, bajo el título «Nuevos Hechos, Nuevo Derecho de la Competencia». El programa de esta jornada está disponible en: https://www.cnmc.es/Portals/0/Ficheros/Home/novedades/competencia/2014/Triptico.pdf 
2. ${ }^{\circ}$ Las Declaraciones de inaplicabilidad, del Artículo 6 LDC, que podrán ser adoptadas, en su caso, por la CNMC, «previo informe del Consejo de Defensa de la Competencia», que como ya señalamos, debe entenderse como preceptivo, aunque no vinculante $\mathrm{y}$,

3. ${ }^{\circ}$ Las Comunicaciones de la CNMC, recogidas en la Disposición Adicional Tercera de la LDC, en la que se precisa que «[e]n particular, las Comunicaciones referentes a los artículos 1 a 3 de esta Ley se publicarán oído el Consejo de Defensa de la Competencia». Esta audiencia, entendemos que, como en el caso anterior, es preceptivo, pero no vinculante para la $\mathrm{CNC}$.

En todos estos casos, sin embargo, el procedimiento prevé únicamente que el CDC será oído, lo que no implica necesariamente, como ocurre con otros órganos en funciones consultivas, que el CDC emita un informe en sentido estricto, en el que pudiera exponer su posición.

Ello nos llevaría a pesar que podría tratarse de un error de base con que se diseñó el CDC: su composición paritaria AGE y Comunidades Autónomas, con el voto de calidad del presidente del CDC, que lo es, el de la CNMC por exigencia legal ${ }^{79}$. Esta fórmula resta eficacia real al órgano, lo que sería especialmente relevante toda vez que sus pronunciamientos carecen de fuerza vinculante. Es decir, el Gobierno o, en su caso, la CNMC puede resolver lo que estime conveniente dentro de sus competencias, sin que sea preciso que el CDC haya siempre de apoyarlo (por el puro reparto de voto), permitiendo dar algún valor a las opiniones contrarias que, en su caso, se pudieran suscitar en su seno.

\section{CONCLUSIONES: HACIA UNA VERDADERA RED ESPAÑOLA DE AUTORIDADES DE COMPETENCIA}

Lo expuesto hasta ahora nos llama a la reflexión. Dos son, al menos, los puntos débiles del llamado Sistema Español de Defensa de la Competencia: el sistema de asignación de casos diseñado sobre la base de «competencias excluyentes» y la inexistencia de una verdadera Red Española de Autoridades de Competencia. Sin embargo, ambos pueden tener solución.

Respecto del primero, el sistema de competencias excluyentes puede mejorarse, si la interpretación constitucional no admite el cambio de fórmula hacia el más eficiente, a nuestro juicio, de las competencias concurrentes.

En este sentido, y como ya tuvimos ocasión de señalar, parecería más razonable admitir una aplicación más «flexible» de este criterio, de tal suerte

79 Vid. Orden ECC/2557/2014, de 29 de diciembre, por la que se nombran los miembros del Pleno del Consejo de Defensa de la Competencia en representación de la Administración General del Estado y su Secretario (BOE Núm. 8, de 9 de enero de 2015). 
que la simple existencia de «desbordamientos» del ámbito territorial autonómico; esto es, de intercambios comerciales con otras zonas del territorio nacional, o la existencia de algún establecimiento fuera de la comunidad autónoma de referencia, no debería generar, por sí solo, una alteración de la libre competencia en un ámbito supra-autonómico en el sentido del artículo 1 de la Ley $1 / 2002$.

Por tanto, nos parece razonable interpretar esta norma en el sentido de que la competencia ejecutiva corresponderá al Estado sólo cuando la conducta restrictiva afecte de forma significativa a un mercado supra-autonómico o al conjunto del mercado nacional.

La vía sería admitir que el desbordamiento debería ser sensible o significativo, concepto ya presente en el ordenamiento español en textos como la Ley 27/1999, de 16 de julio, de cooperativas, que resulta de aplicación a las sociedades cooperativas que desarrollen su actividad cooperativizada en el «territorio de varias Comunidades Autónomas», excepto cuando en una de ellas se desenvuelva «con carácter principal», caso en el que la cooperativa quedaría sometida a la legislación específica de la correspondiente Comunidad Autónoma ${ }^{80}$.

Esta idea se podría combinar con la atribución de una competencia general a la CNMC que podría asumir la competencia de aquellos casos autonómicos cuya importancia o especial complejidad podría superar la capacidad de actuación de la correspondiente autoridad autonómica.

Otra fórmula podría ser, como ya se ha sugerido ${ }^{81}$, tomar como punto de partida la Ley de la Competencia alemana, estableciendo como único punto de conexión el efecto territorial de la conducta (artículo 48.2) pero con un mecanismo de flexibilidad: que permite a la autoridad regional ceder un asunto a la autoridad central (artículo 49.3), y el caso inverso, si ninguna otra autoridad regional se opone (artículo 49.4). Asimismo, y como nos recuerda este mismo autor, los tribunales alemanes exigen que el efecto sea 'sustancial'; término equivalente al de afectación sensible o significativa al que ya aludimos.

${ }^{80}$ Esta interpretación ha sido defendida por COSTAS COMESAÑA, J.: «La descentralización del Derecho español de defensa de la competencia: Una visión crítica de la STC 308/99 y la Ley de coordinación», en el marco del Encuentro sobre el presente y el futuro del sistema comunitario y español de defensa de la competencia, organizado por la Universidad Internacional Menéndez Pelayo, sede de Galicia, celebrado en Pontevedra, los días 12 a 14 de julio de 2006, de quien tomamos esta idea y que consideramos plenamente vigente y asumible.

${ }^{81}$ En este sentido, Berasategi, J., «Defensa de la competencia descentralizada», el Diario Cinco Días, del 19-10-2006. Este breve, pero interesante artículo, cuya contenido desconocemos si ha publicado con mayor detalle en algún otro sitio, puede descargarse en http://cincodias.com/cincodias/2006/10/19/economia/1161370547_850215.html 
Para ello sería preciso modificar la Ley $1 / 2002$, circunstancia que podría aprovecharse para incorporar la nueva redacción a la vigente LDC, aprovechando los huecos que en su articulado ha dejado la aprobación de la Ley 3/2013, de 4 de junio, de creación de la Comisión Nacional de los Mercados y la Competencia. Esta solución técnica implicaría derogar la actual Ley 1/2002 y dotar de pleno contenido a la LDC, al incorporar una cuestión de tanta transcendencia como la relativa al reparto competencia.

El segundo gran tema sería crear una verdadera Red Española de Autoridades de Competencia. En este punto el sistema español es más avanzado que el europeo, aunque sus resultados han demostrado que podría ser más eficiente. El punto de partida sería el Consejo de Defensa de la Competencia, órgano del que carece propiamente la ECN.

Sería necesario reformularlo, tanto en su composición, artificialmente paritaria y con una presidencia ex lege, como en sus funciones y sacar mayor partido a la red virtual CIRCBAC, cuya dimensión pública debería potenciarse y abrirse al público del mismo modo que lo está la ICN o la ECN. Dando ahí la idea de un verdadero sistema integrado de autoridades de competencia españolas que, desde sus respectivas competencias, trabajan en un objetivo común: el fortalecimiento de la competencia en el mercado español.

Esta idea debe presidir también la intervención de la CNMC en los expedientes autonómicos, reemplazando, posiblemente, su consideración de parte interesada por la legitimación para acudir a la vía contenciosa, en caso de desacuerdo, por un sistema de Amiicus Curiae, como ocurre en las relaciones entre la Comisión Europea y las ANC, respecto de los expedientes en los que la autoridad «mejor situada» es la ANC.

El reparto de competencia actual, y ello no tendría porque cambiar, no establece una jerarquía entre la CNMC y las Autoridades Autonómicas de Competencia, sino de igualdad en sus respectivos ámbitos de actuación. De ello se sigue que la única capacidad unificadora, en puridad, procede de los tribunales y no de la CNMC, cuyos criterios, entendemos, no son inamovibles, ni los únicos posibles, por lo que no se deberían excluir criterios alternativos, al menos, mientras los tribunales no se pronuncien al respecto.

De este modo tendríamos una verdadera red, creíble y capaz. Todo es ponerse a ello.

TITTLE: The «Spanish competition authorities network»: the opportunities of a descentralizated system to grow up in efficiency.

RESUMEN: El presente trabajo analiza el papel de las redes de competencia en una eficiente aplicación de las normas antitrust con la perspectiva de lograr un marco normativo de carácter internacional, sin que deba descartarse la posibilidad de llegar al consenso necesario para articular 
una autoridad capaz de resolver eventuales conflictos de dimensión internacional.

Su estudio conjunto nos permitirá no sólo conocerlas mejor, sino aprender de su experiencia, con el propósito de configura del modo más eficiente posible una verdadera Red Española de Autoridades de Competencia.

PALABRAS CLAVE: Derecho de la Competencia, Organización Mundial del Comercio (OMC), Red Internacional de Competencia (RIC), Red Europea de Competencia (REC).

ABSTRACT: This paper analyzes the role of competition networks in an efficient application of antitrust rules with a view to achieving international regulatory framework, without sprading out the possibility of reaching consensus necessary to articulate an authority able to resolve any conflicts with an international dimension.

Their joint study will allow us not only to know them better, but to learn from their experience, in order to set the most efficient manner possible a true Spanish Network of Competition Authorities.

KEY WORDS: Competition Law, antitrust Law, World Trade Organizatrion (WTO), International Competition Network (ICN), European Competition Network (ICN).

RECIBIDO: 15.05 .2015

ACEPTADO: 29.05.2015 
